\title{
Fatal Intestinal Perforation in a Pediatric Neurosurgical Patient
}

\author{
Sangeetha R.P. ${ }^{1} \quad$ Sriganesh Kamath ${ }^{1} \quad$ Dwarakanath Srinivas ${ }^{2} \quad$ Sudhir Venkataramaiah ${ }^{1}$
}

\author{
${ }^{1}$ Department of Neuroanesthesiology and Neurocritical Care, \\ National Institute of Mental Health and Neurosciences, \\ Bengaluru, India \\ 2Department of Neurosurgery, National Institute of Mental Health \\ and Neurosciences, Bengaluru, India
}

J Neuroanaesthesiol Crit Care 2019;6:43-44

Intestinal perforation is rare in neurosurgical population despite risk factors such as surgical stress, concomitant use of nonsteroidal anti-inflammatory drugs (NSAIDs) and corticosteroids, chemotherapy, and infection. We report fatal duodenal perforation in a child and discuss the possible factors contributing to this complication.

A 4-year-old male child weighing $15 \mathrm{~kg}$ underwent re-exploration and decompression of a recurrent ependymoma (-Fig. 1A). The child received cefazolin $400 \mathrm{mg} /$ day, pantoprazole $10 \mathrm{mg} / \mathrm{day}$, and dexamethasone $8 \mathrm{mg} /$ day during the perioperative period. He was operated earlier for a fourth ventricular anaplastic ependymoma at 1 year and had received 6 cycles of chemotherapy and 31 cycles of radiotherapy.

External ventricular drain was placed in view of neurological deterioration secondary to hydrocephalus on the third postoperative day (POD), along with elective ventilation. The child was started on enteral feeds on the second POD and tolerated the same until seventh POD, when the child developed melena (preceded by a decrease in hemoglobin to $9 \mathrm{~g} / \mathrm{dL}$ the previous day) and coffee-ground aspiration with abdominal distension and guarding, suggestive of acute abdomen. Neurological status further worsened with subsequent onset of features of septic shock requiring change in antibiotics and noradrenaline infusion. Emergent treatment was started and one unit of red blood cells was transfused. Abdominal radiograph revealed free air under the diaphragm (-Fig. 1B). An emergent exploratory laparotomy and primary repair were performed followed by peritoneal lavage for duodenal perforation ( - Fig. 1C). Bilateral transverse abdominis plane block was performed for postoperative analgesia to avoid opioids and NSAIDs. However, despite continued supportive measures, the child succumbed 30 hours after surgery to multiorgan dysfunction.

A combination of surgical stress, corticosteroid use, and sepsis is the likely cause for duodenal perforation in this previously asymptomatic child. As steroids can mask inflammatory signs of peritonitis, diagnosis should be considered if patient has abdominal discomfort, fever, leucocytosis,

received

October 1, 2018

accepted after revision

November 15, 2018

published online

January 31, 2019

Address for correspondence Sangeetha R.P., MD, DNB, Department of Neuroanesthesiology and Neurocritical Care, National Institute of Mental Health and Neurosciences, Third floor, Faculty block, Bengaluru-560029, India (e-mail: sangeetharp14@gmail.com).

and melena or gastrointestinal bleeding with guarding and rigidity of abdomen. Abdominal tenderness, the earliest sign of peritonitis, was masked by the poor neurological status, and dexamethasone further delayed its manifestation, leading to sepsis and poor outcome despite an emergency laparotomy. This emphasizes the need for a daily auscultation for adequate bowel sounds in neurocritical care patients, particularly if on prolonged steroids and have a high index of suspicion of an abdominal emergency in the presence of the above-mentioned signs. Mortality is high (85\%) in patients receiving high-dose glucocorticoids. ${ }^{1}$ Hence, strategies for prevention (early enteral nutrition, prophylactic $\mathrm{H} 2$ receptor antagonists, and judicious use of corticosteroids and NSAIDs by daily scrutiny and de-escalation) and prompt recognition of this complication by assessment of abdominal tenderness and radiograph of the abdomen should be adopted early.

There are very few reports of intestinal perforation in neurosurgical population, mostly subsequent to ventriculoperitoneal shunt surgery. ${ }^{2}$ Perforation due to NSAIDs or corticosteroids is extremely rare. One study documented intestinal perforation in 5/719 neurosurgical patients who received steroids as compared with none in 3,749 patients who did not receive steroids. Age $>50$ years and pre-existing diverticular disease were identified as risk factors for perforation. ${ }^{3}$ In pediatric population, fatal hemorrhage from duodenal perforation 10 days after surgery was described in a 10-year-old child with medulloblastoma receiving highdose dexamethasone. ${ }^{4}$ Authors of a recent report of two cases of brain stem anaplastic ependymomas attribute duodenal ulceration to autonomic dysfunction-induced gut ischemia, gastric hypersecretion, and adrenal cortical hormonesinduced decreased gastric mucus secretion. ${ }^{5}$ Perforation can complicate 2 to $14 \%$ of duodenal ulcers in general population, ${ }^{6}$ contributing to approximately one-tenth of all acute abdomen diagnosis in medical intensive care units. ${ }^{7}$

Despite perioperative steroids being routinely used in the perioperative period for brain tumors, literature search reveals that guidelines for optimal treatment duration of steroid therapy are lacking. A recent cohort study of 131 newly diagnosed

License terms

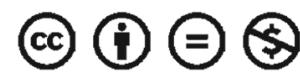




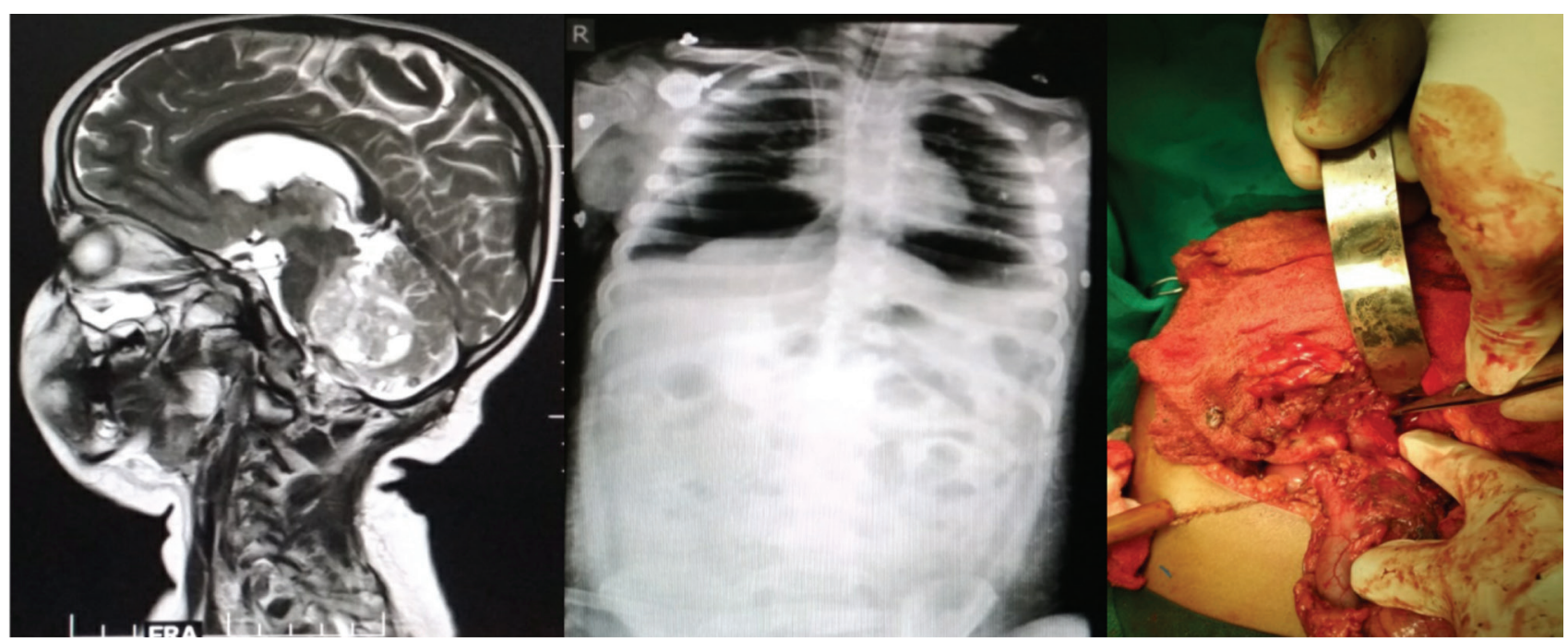

Fig. 1 (A) Recurrent posterior fossa tumor; (B) Radiographic image of free air under the diaphragm; (C) Exploratory laparotomy revealing duodenal perforation.

neurosurgical patients with glioblastoma provides evidence of scope for successful steroid tapering within 2 weeks after surgery with improved survival rates. ${ }^{8}$

To conclude, steroids can mask the symptoms of peritonitis and hence close monitoring and high degree of suspicion are essential. Perioperative steroids should be tapered and discontinued as soon as possible. Safer alternatives to NSAIDs such as paracetamol should be preferred for perioperative analgesia in patients receiving steroids.

\section{Funding}

None.

\section{Conflict of Interest}

None declared.

\section{References}

1 ReMine SG, Mcllrath DC. Bowel perforation in steroid-treated patients. Ann Surg 1980;192(4):581-586

2 Sathyanarayana S, Wylen EL, Baskaya MK, Nanda A. Spontaneous bowel perforation after ventriculoperitoneal shunt surgery: case report and a review of 45 cases. Surg Neurol 2000;54(5):388-396
3 Weiner HL, Rezai AR, Cooper PR. Sigmoid diverticular perforation in neurosurgical patients receiving high-dose corticosteroids. Neurosurgery 1993;33(1):40-43

4 Wong V, Lefloch N, Crawford JR. Fatal gastrointestinal hemorrhage in a young boy with newly diagnosed metastatic medulloblastoma on high dose dexamethasone. Case Rep Pediatr 2014;2014:478326

5 Nobori C, Kimura K, Ohira G, et al. Giant duodenal ulcers after neurosurgery for brainstem tumors that required reoperation for gastric disconnection: a report of two cases. BMC Surg 2016;16(1):75

6 Chung KT, Shelat VG. Perforated peptic ulcer - an update. World J Gastrointest Surg 2017;9(1):1-12

7 Gajic O, Urrutia LE, Sewani H, Schroeder DR, Cullinane DC, Peters SG. Acute abdomen in the medical intensive care unit. Crit Care Med 2002;30(6):1187-1190

8 Díez Valle R, Becerra Castro V, Marigil Sánchez M, Gállego Pérez-Larraya J, Núñez-Córdoba JM, Tejada Solis S. Results of a policy of fast tapering of steroids after resection surgery in glioblastoma. World Neurosurg 2018;109:e845-e852 